\title{
ON PERFECT POWERS WHICH ARE SUM OR DIFFERENCE OF TWO LUCAS NUMBERS
}

\author{
Z. ŞİAR AND R. KESKIN
}

Received 13 February, 2019

\begin{abstract}
In this paper, we consider the Diophantine equation $L_{n} \pm L_{m}=k x^{2}$ with $k \in\{1,2\}$ and

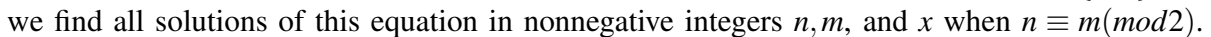
With the help of these solutions, we solve the equation $L_{n}-L_{m}=2^{a}$. In order to solve the last equation, we also use lower bounds for linear forms in logarithms and a version of the BakerDavenport reduction method in diophantine approximation.
\end{abstract}

2010 Mathematics Subject Classification: 11B39, 11J86, 11D61

Keywords: Fibonacci and Lucas numbers, Exponential equations, Linear forms in logarithms, Baker's method

\section{INTRODUCTION}

The Fibonacci sequence $\left(F_{n}\right)$ is defined as $F_{0}=0, F_{1}=1$, and $F_{n}=F_{n-1}+F_{n-2}$ for $n \geq 2$. The Lucas sequence $\left(L_{n}\right)$, which is similar to the Fibonacci sequence, is defined by the same recursive pattern with initial conditions $L_{0}=2, L_{1}=1$. Binet's formulas

$$
F_{n}=\frac{\alpha^{n}-\beta^{n}}{\sqrt{5}}
$$

and

$$
L_{n}=\alpha^{n}+\beta^{n}
$$

are well known, where $\alpha=\frac{1+\sqrt{5}}{2}$ and $\beta=\frac{1-\sqrt{5}}{2}$, which are the roots of the characteristic equation $x^{2}-x-1=0$. It can be seen that $1<\alpha<2$ and $-1<\beta<0$. The following relations given for Fibonacci and Lucas numbers will be useful for us:

$$
\begin{gathered}
\alpha^{n-1} \leq L_{n} \leq 2 \alpha^{n} \text { for } n \geq 0, \\
\left(F_{m}, F_{n}\right)=F_{(m, n)}, \\
L_{n+m}+L_{n-m}=\left\{\begin{array}{cc}
5 F_{m} F_{n} & \text { if } n \text { is odd }, \\
L_{n} L_{m} & \text { otherwise. }
\end{array}\right.
\end{gathered}
$$


and

$$
L_{n+m}-L_{n-m}=\left\{\begin{array}{c}
L_{n} L_{m} \text { if } n \text { is odd, } \\
5 F_{m} F_{n} \text { otherwise. }
\end{array} .\right.
$$

The relations (1.1), (1.3), and (1.4) can be proved using Binet's formulae. The Fibonacci and Lucas sequences are the most important among the second order linear recussive sequences and have been investigated by many researchers. The problem to find all perfect powers in the Fibonacci sequence and in the Lucas sequence have been an interesting problem for many years. Finally, this problem was solved by Bugeaud, Mignotte and Siksek in [8]. They stated that the only perfect powers in the Fibonacci sequence are $0,1,8,144$ and the only perfect powers in the Lucas sequence are 1 and 4. Then, in [7], the authors found all the nonnegative integer solutions $(n, y, p)$ of the equations

$$
F_{n} \pm 1=y^{p}, p \geq 2 .
$$

Later, in [12], Luca and Patel have tackled the problem to find the integer solutions $(n, m, y, p)$ of the Diophantine equation

$$
F_{n} \pm F_{m}=y^{p}, p \geq 2 .
$$

They found that if $n \equiv m(\bmod 2)$, this equation has solution either $\max \{|n|,|m|\} \leq 36$ or $y=0$ and $|n|=|m|$.

Recently, many mathematicians, in [2-5], have handled some exponential Diophantine equations such as

$$
u_{n}+u_{m}=2^{a} \text { and } u_{n}+u_{m}+u_{r}=2^{a},
$$

where $\left(u_{n}\right)$ is the Fibonacci sequence or the Lucas sequence or the Pell sequence. Furthermore, In [15] , we determined the nonnegative integer solutions $(n, m, a)$ of the equation $F_{n}-F_{m}=2^{a}$. In this study, we solve the equation $L_{n} \pm L_{m}=k x^{2}$ with $k=1,2$ when $n \equiv \operatorname{m(\operatorname {mod}2)}$. With help of these solutions, and motivated by the studies of Bravo and Luca [4], we find all nonnegative integers solutions $(n, m, a)$ of the equation

$$
L_{n}-L_{m}=2^{a} .
$$

In this paper, we follow the approach and the method presented in [4]. Therefore, in section 2, we introduce necessary lemmas and theorems related to this method. Then in section 3 , we prove our main theorems.

\section{Auxiliary RESUlts}

Lately, in many articles, to solve Diophantine equations such as the equation (1.6), authors have used Baker's theory lower bounds for a nonzero linear form in logarithms of algebraic numbers. Since such bounds are of crucial importance in effectively solving of Diophantine equations, we start with recalling some basic notions from algebraic number theory. 
Let $\eta$ be an algebraic number of degree $d$ with minimal polynomial

$$
a_{0} x^{d}+a_{1} x^{d-1}+\ldots+a_{d}=a_{0} \prod_{i=1}^{d}\left(X-\eta^{(i)}\right) \in \mathbb{Z}[x],
$$

where the $a_{i}$ 's are relatively prime integers with $a_{0}>0$ and $\eta^{(i)}$ 's are conjugates of $\eta$. Then

$$
h(\eta)=\frac{1}{d}\left(\log a_{0}+\sum_{i=1}^{d} \log \left(\max \left\{\left|\eta^{(i)}\right|, 1\right\}\right)\right)
$$

is called the logarithmic height of $\eta$. In particularly, if $\eta=a / b$ is a rational number with $\operatorname{gcd}(a, b)=1$ and $b>1$, then $h(\eta)=\log (\max \{|a|, b\})$.

The following properties of logarithmic height are found in many works stated in references:

$$
\begin{gathered}
h(\eta \pm \gamma) \leq h(\eta)+h(\gamma)+\log 2 \\
h\left(\eta \gamma^{ \pm 1}\right) \leq h(\eta)+h(\gamma), \\
h\left(\eta^{s}\right)=|s| h(\eta) .
\end{gathered}
$$

The following theorem, is deduced from Corollary 2.3 of Matveev [13], provides a large upper bound for the subscript $n$ in the equation (1.6) (also see Theorem 9.4 in [8]).

Theorem 1. Assume that $\gamma_{1}, \gamma_{2}, \ldots, \gamma_{t}$ are positive real algebraic numbers in a real algebraic number field $\mathbb{K}$ of degree $D, b_{1}, b_{2}, \ldots, b_{t}$ are rational integers, and

$$
\Lambda:=\gamma_{1}^{p_{1}} \ldots \gamma_{t}^{p_{t}}-1
$$

is not zero. Then

$$
|\Lambda|>\exp \left(-1.4 \cdot 30^{t+3} \cdot t^{4.5} \cdot D^{2}(1+\log D)(1+\log B) A_{1} A_{2} \ldots A_{t}\right),
$$

where

$$
B \geq \max \left\{\left|b_{1}\right|, \ldots,\left|b_{t}\right|\right\},
$$

and $A_{i} \geq \max \left\{D h\left(\gamma_{i}\right),\left|\log \gamma_{i}\right|, 0.16\right\}$ for all $i=1, \ldots, t$.

The following lemma, was proved by Dujella and Pethô [10], is a variation of a lemma of Baker and Davenport [1]. And this lemma will be used to reduce the upper bound for the subscript $n$ in the equation (1.6). In the following lemma, the function $\|\cdot\|$ denotes the distance from $x$ to the nearest integer. That is, $\|x\|=$ $\min \{|x-n|: n \in \mathbb{Z}\}$ for a real number $x$.

Lemma 1. Let $M$ be a positive integer, let $p / q$ be a convergent of the continued fraction of the irrational number $\gamma$ such that $q>6 M$, and let $A, B, \mu$ be some real numbers with $A>0$ and $B>1$. Let $\varepsilon:=\|\mu q\|-M\|\gamma q\|$. If $\varepsilon>0$, then there exists no solution to the inequality

$$
0<|u \gamma-v+\mu|<A B^{-w},
$$


in positive integers $u, v$, and $w$ with

$$
u \leq M \text { and } w \geq \frac{\log (A q / \varepsilon)}{\log B} .
$$

From (1.3) and (1.4), we can give the following lemma.

Lemma 2. Assume that $n \equiv m(\bmod 2)$. Then

$$
L_{n}+L_{m}=\left\{\begin{array}{c}
5 F_{(n+m) / 2} F_{(n-m) / 2} \text { if } n \equiv m+2(\bmod 4) \\
L_{(n+m) / 2} L_{(n-m) / 2} \text { if } n \equiv m(\bmod 4)
\end{array}\right.
$$

and

$$
L_{n}-L_{m}=\left\{\begin{array}{c}
L_{(n+m) / 2} L_{(n-m) / 2} \text { if } n \equiv m+2(\bmod 4) \\
5 F_{(n+m) / 2} F_{(n-m) / 2} \text { if } n \equiv m(\bmod 4)
\end{array}\right.
$$

The following two theorems and lemma are given in [9] and [11].

Theorem 2. If $F_{n}=x^{2}$, then $n=0,1,2,12$. If $F_{n}=2 x^{2}$, then $n=0,3,6$. If $L_{n}=x^{2}$, then $n=1,3$ and if $L_{n}=2 x^{2}$, then $n=0,6$.

Theorem 3. Let $m>3$ be an integer and $F_{n}=F_{m} x^{2}$ for some $x \in \mathrm{Z}$. Then $n=m$.

Lemma 3. There is no integer $x$ such that $F_{n}=5 F_{m} x^{2}$ for $m \geq 3$.

The proof of following theorem is similar to that of the above lemma. Therefore, we omit its proof.

Theorem 4. There is no integer $x$ such that $F_{n}=10 F_{m} x^{2}$ for $m \geq 1$.

From Theorem 4.1 and Theorem 4.2 given in [14], we can deduce the following results.

Theorem 5. If $L_{n} L_{m}=x^{2}$ for $1 \leq m \leq n$, then $n=m$ or $(n, m)=(3,1)$.

Theorem 6. If $L_{n} L_{m}=2 x^{2}$ for $1 \leq m \leq n$, then $(n, m)=(3,1),(6,1)$, or $(6,3)$.

\section{MAIN THEOREMS}

Theorem 7. Let $m \leq n$ and $n \equiv m(\bmod 2)$. Then all solutions of the equation $L_{n}+$ $L_{m}=k x^{2}$ with $k=1,2$ in nonnegative integers $n, m$, are given by

$$
(n, m, x)=(6,6,6),(17,7,60),(6,4,5), \text { and }\left(4 r, 0, L_{2 r}\right) \text { with } r \geq 0
$$

when $k=1$, and

$$
(n, m, x)=(1,1,1),(3,3,2),(11,1,10), \text { and }(8,2,5)
$$

when $k=2$. 
Proof. Assume that $n \equiv m(\bmod 2)$. If $n=m$, we have $L_{n}=2 z^{2}$ for $k=1$, which implies that $n=0,6$, or $L_{n}=x^{2}$ for $k=2$, which implies that $n=1,3$ by Theorem 2 . Then, assume that $n>m$.

Firstly, let $n \equiv m+2(\bmod 4)$. Then we can write $5 F_{(n+m) / 2} F_{(n-m) / 2}=k x^{2}$ by Lemma 2. Thus we get the equations $F_{(n+m) / 2}=a F_{d} u^{2}, F_{(n-m) / 2}=b F_{d} v^{2}$ for $k=1$ and $F_{(n+m) / 2}=a F_{d} u^{2}, F_{(n-m) / 2}=2 b F_{d} v^{2}$ or $F_{(n+m) / 2}=2 a F_{d} u^{2}, F_{(n-m) / 2}=b F_{d} v^{2}$ for $k=2$, where $a b=5$ and $\left(F_{(n+m) / 2}, F_{(n-m) / 2}\right)=F_{d}$ by (1.2). Assume that $a=1, b=5$. Then we have $F_{(n+m) / 2}=u^{2}, F_{(n-m) / 2}=5 v^{2}$ for $k=1$ by Lemma 3, and $F_{(n+m) / 2}=$ $2 F_{d} u^{2}, F_{(n-m) / 2}=5 F_{d} v^{2}$ for $k=2$ by Theorem 4. If $F_{(n+m) / 2}=u^{2}, F_{(n-m) / 2}=$ $5 v^{2}=F_{5} v^{2}$, then it can be easily seen that $n=17$ and $m=7$ by Theorems 2 and 3. If $F_{(n+m) / 2}=2 F_{d} u^{2}, F_{(n-m) / 2}=5 F_{d} v^{2}$, then $d \leq 2$ by Lemma 3, i.e., $F_{(n+m) / 2}=$ $2 u^{2}, F_{(n-m) / 2}=5 v^{2}$. Thus we get $n=11$ and $m=1$ by Theorems 2 and 3 . Assume that $a=5$ and $b=1$. Then we have the equation $F_{(n+m) / 2}=5 u^{2}, F_{(n-m) / 2}=v^{2}$ for $k=1$ by Lemma 3, and $F_{(n+m) / 2}=5 F_{d} u^{2}, F_{(n-m) / 2}=2 F_{d} v^{2}$ for $k=2$ by Theorem 4 . Similarly, it can be seen that $n=6, m=4$ for $k=1$ and $n=8, m=2$ for $k=2$.

Secondly, let $n \equiv m(\bmod 4)$. Then we can write $L_{(n+m) / 2} L_{(n-m) / 2}=k x^{2}$ by Lemma 2. Using Theorems 5 and 6 , we get $m=0$ or $n=4, m=2$ for $k=1$, and $(n, m)=$ $(4,2),(7,5),(9,3)$ for $k=2$. If $m=0$, then $n \equiv 0(\bmod 4)$. This implies that $n=4 r$ for some integer $r$. Besides, if $m=0$, we have $x^{2}=L_{n}+2=L_{4 r}+2=L_{2 r}^{2}$. Hence $\left(4 r, 0, L_{2 r}\right)$ is a solution of the equation $L_{n}+L_{m}=x^{2}$. Also, it is clear that $L_{4}+L_{2} \neq$ $k x^{2}$ for $k=1,2$, and $L_{7}+L_{5} \neq 2 x^{2}, L_{9}+L_{3} \neq 2 x^{2}$.

Since the proof of the following theorem is similar, we omit its proof.

Theorem 8. Let $n \equiv m(\bmod 2)$. Then all solutions of the equation $L_{n}-L_{m}=k x^{2}$ with $k=1,2$, in nonnegative integers $n, m$, and $x$, are given by

$$
(n, m, x)=(4,2,2),(7,3,5) \text { or }\left(4 r+2,0, L_{2 r+1}\right) \text { with } r \geq 0
$$

when $k=1$, and

when $k=2$.

$$
(n, m, x)=(7,5,3),(9,3,6)
$$

The following lemma, which will be used in the next theorem, gives a sufficient condition for a rational number to be a convergent of a given real number.

Lemma 4. ([6])Let $\gamma$ be a real number. Any non-zero rational number $\frac{a}{b}$ with

$$
\left|\gamma-\frac{a}{b}\right|<\frac{1}{2 b^{2}}
$$

is a convergent of $\gamma$.

Theorem 9. The only solutions of the Diophantine equation $L_{n}-L_{m}=2^{a}$ in nonnegative integers $m<n$, and $a$, are given by

$$
(n, m, a) \in\{(2,0,0),(3,0,1),(6,0,4),(2,1,1)\}
$$


and

$$
(n, m, a) \in\{(3,2,0),(4,2,2),(5,2,3),(5,4,2)\} .
$$

Proof. Assume that the equation $L_{n}-L_{m}=2^{a}$ holds in nonnegative integers $m<$

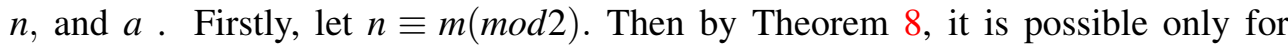
$\left(n, m, 2^{a / 2}\right)=(4,2,2)$, which implies that $(n, m, a)=(4,2,2)$ or $\left(n, m, 2^{a / 2}\right)=(4 r+$ $\left.2,0, L_{2 r+1}\right)$. This yields to $(n, m, a)=(2,0,0),(6,0,4)$. Secondly, let $n \not \equiv m(\bmod 2)$. If $n-m=1$, then we get the equation $L_{n-2}=2^{a}$. This equation implies that $(n, m, a)=$ $(3,2,0),(5,4,2),(2,1,1)$ by Theorem 2 . If $n-m=3$, then we have the equation $L_{n-2}=2^{a-1}$, which shows that $(n, m, a)=(3,0,1),(5,2,3)$ by Theorem 2 . Thus we may assume that $n-m \geq 5$. With the help of Mathematica program, one can see that the equation (1.6) has no solutions for $0 \leq m<n \leq 200$ and $n-m \geq 5$. From now on, assume that $n>200$ and $n-m \geq 5$. Now, let us show that $a<n$. Using (1.1), we get the inequality

$$
2^{a}=L_{n}-L_{m}<L_{n} \leq 2 \alpha^{n}<2^{n+1},
$$

which yields to $a \leq n$.

On the other hand, rearranging the equation (1.6) as $\alpha^{n}-2^{a}=L_{m}-\beta^{n}$ and taking absolute values, we obtain

$$
\left|\alpha^{n}-2^{a}\right|=\left|L_{m}-\beta^{n}\right| \leq L_{m}+|\beta|^{n}<2 \alpha^{m}+\frac{1}{2}
$$

by (1.1). If we divide both sides of the above inequality by $\alpha^{n}$, we get

$$
\left|1-2^{a} \alpha^{-n}\right|<\frac{3}{\alpha^{n-m}}
$$

where we have used the facts that $\frac{1}{2} \alpha^{-m}<1$ and $n>m$. Now, let us apply Theorem 1 with $\gamma_{1}:=2, \gamma_{2}:=\alpha$ and $b_{1}:=a, b_{2}:=-n$. Note that the numbers $\gamma_{1}$ and $\gamma_{2}$ are positive real numbers and elements of the field $K=Q(\sqrt{5})$, so $D=2$. It can be shown that the number $\Lambda_{1}:=2^{a} \alpha^{-n}-1$ is nonzero. Indeed, if $\Lambda_{1}=0$, then we get

$$
2^{a}=\alpha^{n}=L_{n}-\beta^{n}>L_{n}-1 \geq L_{n}-L_{m}=2^{a},
$$

which is impossible. Since $h\left(\gamma_{1}\right)=\log 2=0.6931 \ldots$ and $h\left(\gamma_{2}\right)=\frac{\log \alpha}{2}=\frac{0.4812 \ldots}{2}$ by (2.1), we can take $A_{1}:=1.4, A_{2}:=0.5$. Also, since $a \leq n$, we can take $B:=$ $\max \{|a|,|-n|, 1\}=n$. Thus, taking into account the inequality (3.1) and using Theorem 1 , we obtain

$$
\frac{3}{\alpha^{n-m}}>\left|\Lambda_{1}\right|>\exp \left(-1.4 \cdot 30^{5} \cdot 2^{4.5} \cdot 2^{2}(1+\log 2)(1+\log n)(1.4)(0.5)\right)
$$

and so

$$
(n-m) \log \alpha-\log 3<1.4 \cdot 30^{5} \cdot 2^{4.5} \cdot 2^{2}(1+\log 2)(1+\log n)(1.4)(0.5)
$$


Now, we try to apply Theorem 1 a second time. Rearranging the equation (1.6) as $\alpha^{n}-\alpha^{m}-2^{a}=-\beta^{n}+\beta^{m}$ and taking absolute values in here, we obtain

$$
\left|\alpha^{n}\left(1-\alpha^{m-n}\right)-2^{a}\right|=\left|-\beta^{n}+\beta^{m}\right| \leq|\beta|^{n}+|\beta|^{m}<4 / 3,
$$

where we used the fact that $|\beta|^{n}+|\beta|^{m}<4 / 3$ for $n>200$ and $m \geq 0$. Dividing both sides of the above inequality by $\alpha^{n}\left(1-\alpha^{m-n}\right)$, we get

$$
\left|1-2^{a} \alpha^{-n}\left(1-\alpha^{m-n}\right)^{-1}\right|<\frac{4 / 3}{\alpha^{n}\left(1-\alpha^{m-n}\right)} .
$$

Since

it is seen that

$$
\alpha^{m-n}=\frac{1}{\alpha^{n-m}}<\frac{1}{\alpha}<\frac{2}{3}
$$

$$
1-\alpha^{m-n}>1-\frac{2}{3}=\frac{1}{3}
$$

and therefore

$$
\frac{1}{1-\alpha^{m-n}}<3
$$

Then from (3.3), it follows that

$$
\left|1-2^{a} \alpha^{-n}\left(1-\alpha^{m-n}\right)^{-1}\right|<\frac{4}{\alpha^{n}} .
$$

Thus, taking $\gamma_{1}:=2, \gamma_{2}:=\alpha, \gamma_{3}:=\left(1-\alpha^{m-n}\right)^{-1}$ and $b_{1}:=a, b_{2}:=-n, b_{3}:=1$, we can apply Theorem 1. One can see that the numbers $\gamma_{1}, \gamma_{2}$, and $\gamma_{3}$ are positive real numbers and elements of the field $K=Q(\sqrt{5})$, so $D=2$. Put $\Lambda_{2}:=2^{a} \alpha^{-n}(1-$ $\left.\alpha^{m-n}\right)^{-1}-1$. Since

$$
\alpha^{n}\left(1-\alpha^{m-n}\right)=\alpha^{n}-\alpha^{m}=L_{n}-\beta^{n}-L_{m}+\beta^{m} \neq 2^{a}
$$

for $n>m$, the number $\Lambda_{2}:=2^{a} \alpha^{-n}\left(1-\alpha^{m-n}\right)^{-1}-1$ is nonzero. Also, since $h\left(\gamma_{1}\right)=$ $\log 2=0.6931 \ldots$, and $h\left(\gamma_{2}\right)=\frac{\log \alpha}{2}=\frac{0.4812 \ldots}{2}$ by (2.1), we can take $A_{1}:=1.4$ and $A_{2}:=0.5$. Besides, using (2.2), (2.3), and (2.4), we get that $h\left(\gamma_{3}\right) \leq \log 2+$ $(n-m) \frac{\log \alpha}{2}$. A simple computation shows that $\left|\log \gamma_{3}\right| \leq \log 4+(n-m) \log \alpha$. So we can take $A_{3}:=\log 4+(n-m) \log \alpha$. Also, since $a \leq n$, it follows that $B:=$ $\max \{|a|,|-n|, 1\}=n$. Thus, taking into account the inequality (3.4) and using Theorem 1 , we obtain

or

$$
\frac{4}{\alpha^{n}}>\left|\Lambda_{2}\right|>\exp (-C)(1+\log 2)(1+\log n)(1.4)(0.5)(\log 4+(n-m) \log \alpha)
$$

$$
n \log \alpha-\log 4<C(1+\log 2)(1+\log n)(1.4)(0.5)(\log 4+(n-m) \log \alpha),
$$

where $C=1.4 \cdot 30^{6} \cdot 3^{4.5} \cdot 2^{2}$. Inserting the inequality (3.2) into the last inequality, a computer search with Mathematica gives us that $n<1.81 \cdot 10^{25}$. 
Now, let us try to reduce the upper bound on $n$ applying Lemma 1. Let

$$
z_{1}:=a \log 2-n \log \alpha .
$$

Then

by (3.1). The inequality

$$
\left|1-e^{z_{1}}\right|<\frac{3}{\alpha^{n-m}}
$$

$$
\alpha^{n}=L_{n}-\beta^{n}>L_{n}-1 \geq L_{n}-L_{m}=2^{a}
$$

implies that $z_{1}<0$. In that case, since $\frac{3}{\alpha^{n-m}}<\frac{1}{2}$ for $n-m \geq 5$, it follows that $e^{\left|z_{1}\right|}<2$. Hence, we get

$$
0<\left|z_{1}\right|<e^{\left|z_{1}\right|}-1=e^{\left|z_{1}\right|}\left|1-e^{z_{1}}\right|<\frac{6}{\alpha^{n-m}},
$$

or

$$
0<|a \log 2-n \log \alpha|<\frac{6}{\alpha^{n-m}} .
$$

Dividing this inequality by $\log \alpha$ and $a$, we get

$$
0<\left|\frac{\log 2}{\log \alpha}-\frac{n}{a}\right|<\frac{13}{a \cdot \alpha^{n-m}} .
$$

Assume that $n-m \geq 137$. Then it can be seen that $\frac{\alpha^{n-m}}{26}>1.6 \cdot 10^{27}>n \geq a$, and so we have $\left|\frac{\log 2}{\log \alpha}-\frac{n}{a}\right|<\frac{13}{a \cdot \alpha^{n-m}}<\frac{1}{2 a^{2}}$. This implies by Lemma 4 that the rational number $\frac{n}{a}$ is a convergent to $\gamma=\frac{\log 2}{\log \alpha}$. Now let $\left[a_{0}, a_{1}, a_{2}, \ldots\right]=[1,2,3,1,2,3,2,4, \ldots]$ be the continued fraction expression of $\gamma$ and let $\frac{p_{k}}{q_{k}}$ be its $k$ th convergent. Assume that $\frac{n}{a}=\frac{p_{t}}{q_{t}}$ for some $t$. Then we have $1.88 \cdot 10^{25}>q_{56}>1.81 \cdot 10^{25}>a$. Thus $t \in\{0,1,2, \ldots, 55\}$. Let $a_{M}=\max \left\{a_{i} \mid i=0,1,2, \ldots, 56\right\}$. Then we find that $a_{M}=134$. From the known properties of continued fraction, we know that

$$
\left|\gamma-\frac{p_{t}}{q_{t}}\right|=\frac{1}{\left(\gamma_{t+1} q_{t}+q_{t-1}\right) q_{t}}=\frac{1}{\left(\gamma_{t+1}+\frac{q_{t-1}}{q_{t}}\right) q_{t}^{2}}>\frac{1}{\left(a_{t+1}+2\right) q_{t}^{2}}>\frac{1}{\left(a_{M}+2\right) q_{t}^{2}}
$$

and this shows that

$$
\left|\gamma-\frac{p_{t}}{q_{t}}\right|>\frac{1}{\left(a_{M}+2\right) a^{2}}
$$

where we have used the facts that $a_{t}=\left\lfloor\gamma_{t}\right\rfloor$ and $q_{t-1}<q_{t}$. Thus, from (3.6), we obtain

$$
\frac{13}{a \cdot \alpha^{n-m}}>\frac{1}{\left(a_{M}+2\right) a^{2}},
$$


which implies that

$$
\frac{1}{3.2 \cdot 10^{27}}>\frac{13}{\alpha^{n-m}}>\frac{1}{136 \cdot q_{56}}>\frac{1}{2.55 \cdot 10^{27}},
$$

a contradiction. Therefore $n-m<137$. Substituting this upper bound for $n-m$ into (3.5), we obtain $n<3.5 \cdot 10^{15}$.

Now, let

$$
z_{2}:=a \log 2-n \log \alpha-\log \left(\left(1-\alpha^{m-n}\right)\right) .
$$

In this case,

$$
\left|1-e^{z_{2}}\right|<\frac{4}{\alpha^{n}}
$$

by (3.4). It is seen that $\frac{4}{\alpha^{n}}<\frac{1}{2}$. If $z_{2}>0$, then $0<z_{2}<e^{z_{2}}-1<\frac{4}{\alpha^{n}}$. If $z_{2}<0$, then $\left|1-e^{z_{2}}\right|=1-e^{z_{2}}<\frac{4}{\alpha^{n}}<\frac{1}{2}$. From this, we get $e^{\left|z_{2}\right|}<2$ and therefore

$$
0<\left|z_{2}\right|<e^{\left|z_{2}\right|}-1=e^{\left|z_{2}\right|}\left|1-e^{z_{2}}\right|<\frac{8}{\alpha^{n}} .
$$

In both case, the inequality

is true. That is,

$$
0<\left|z_{2}\right|<\frac{8}{\alpha^{n}}
$$

$$
0<\left|a \log 2-n \log \alpha-\log \left(1-\alpha^{m-n}\right)\right|<\frac{8}{\alpha^{n}} .
$$

Dividing both sides of the above inequality by $\log \alpha$, we get

$$
0<\left|a\left(\frac{\log 2}{\log \alpha}\right)-n+\frac{\log \left(1-\alpha^{m-n}\right)^{-1}}{\log \alpha}\right|<17 \cdot \alpha^{-n} .
$$

Let $\gamma:=\frac{\log 2}{\log \alpha}$ and $M:=3.5 \cdot 10^{15}$. Then the denominator of the $63^{\text {rd }}$ convergent of $\gamma$ exceeds $6 M$. Also, taking

$$
\mu:=\frac{\log \left(1-\alpha^{m-n}\right)^{-1}}{\log \alpha}
$$

a quick computation using Mathematica gives us that

$$
\varepsilon: \varepsilon(\mu)=\|\mu q\|-M\|\gamma q\|>0
$$

for every odd $n-m \in[5,137]$. Let $A:=17, B:=\alpha$, and $w:=n$ in Lemma 1 . Then, it follows from Lemma 1, applied to the inequality (3.7) in order to reduce a little bit the upper bound on $n$, that

$$
n<\frac{\log \left(A q_{64} / \varepsilon\right)}{\log B} \leq 155 .
$$

Thus we can say that if $(n, m, a)$ is a solution of the equation (1.6), then $n<155$. This contradicts our assumption that $n>200$. This completes the proof. 


\section{REFERENCES}

[1] A. Baker and H. Davenport, "The equations $3 x^{2}-2=y^{2}$ and $8 x^{2}-7=z^{2}$." Q. J. Math., Oxf. II. Ser., vol. 20, no. 1, pp. 129-137, 1969, doi: 10.1093/qmath/20.1.129.

[2] E. F. Bravo and J. J. Bravo, "Powers of two as sums of three Fibonacci numbers." Lith. Math. J., vol. 55, no. 3, pp. 301-311, 2015, doi: 10.1007/s10986-015-9282-z.

[3] J. J. Bravo, B. Faye, and F. Luca, "Powers of two as sums of three Pell numbers." Taiwanese J. Math., vol. 21, no. 4, pp. 739-751, 2017, doi: 10.11650/tjm/7840.

[4] J. J. Bravo and F. Luca, "Powers of Two as Sums of Two Lucas Numbers." J. Integer Seq., vol. 17, no. 8, pp. article 14.8.3, 12, 2014.

[5] J. J. Bravo and F. Luca, "On the Diophantine Equation $F_{n}+F_{m}=2^{a}$." Quaest. Math., vol. 39, no. 3, pp. 391-400, 2016, doi: 10.2989/16073606.2015.1070377.

[6] Y. Bugeaud, Linear forms in logarithms and applications. Zürich: European Mathematical Society (EMS), 2018, vol. 28, doi: 10.4171/183.

[7] Y. Bugeaud, F. Luca, M. Mignotte, and S. Siksek, "Fibonacci numbers at most one away from a perfect power." Elem. Math., vol. 63, no. 2, pp. 65-75, 2008, doi: 10.4171/EM/89.

[8] Y. Bugeaud, M. Mignotte, and S. Siksek, "Classical and modular approaches to exponential Diophantine equations. I: Fibonacci and Lucas perfect powers." Ann. Math. (2), vol. 163, no. 3, pp. 969-1018, 2006, doi: 10.4007/annals.2006.163.969.

[9] J. H. E. Cohn, "Square Fibonacci numbers, etc.” Fibonacci Q., vol. 2, pp. 109-113, 1964.

[10] A. Dujella and A. Pethő, "A generalization of a theorem of Baker and Davenport." Q. J. Math., Oxf. II. Ser., vol. 49, no. 195, pp. 291-306, 1998, doi: 10.1093/qjmath/49.195.291.

[11] R. Keskin and Z. Yosma, "On Fibonacci and Lucas numbers of the form $c x^{2}$." J. Integer Seq., vol. 14, no. 9, pp. article 11.9.3, 12, 2011.

[12] F. Luca and V. Patel, "On perfect powers that are sums of two Fibonacci numbers." J. Number Theory, vol. 189, pp. 90-96, 2018, doi: 10.1016/j.jnt.2018.02.003.

[13] E. M. Matveev, "An explicit lower bound for a homogeneous rational linear form in logarithms of algebraic numbers. II." Izv. Math., vol. 64, no. 6, pp. 1217-1269, 2000, doi: 10.1070/IM2000v064n06ABEH000314.

[14] Z. Şiar, "On square classes in generalized Lucas sequences." Int. J. Number Theory, vol. 11, no. 2, pp. 661-672, 2015, doi: 10.1142/S1793042115500414.

[15] Z. Şiar and R. Keskin, "On the Diophantine equation $F_{n}-F_{m}=2^{a}$." Colloquium Mathematicum, vol. 159, no. 1, pp. 119-126, 2020, doi: 10.4064/cm7485-12-2018.

Authors' addresses

Z. Şiar

(Corresponding author) Bingöl University, Department of Mathematics, Bingöl, Turkey

E-mail address: zsiar@bingol.edu.tr

\section{R. Keskin}

Sakarya University, Department of Mathematics, Sakarya, Turkey

E-mail address: rkeskin@sakarya.edu.tr 